QUARTERLY OF APPLIED MATHEMATICS

VOLUME LXVIII, NUMBER 1

MARCH 2010, PAGES 61-71

S 0033-569X(09)01178-1

Article electronically published on October 20, 2009

\title{
HIGH FREQUENCY SOLUTIONS OF THE NONLINEAR SCHRÖDINGER EQUATION ON SURFACES
}

\author{
BY \\ NICOLAS BURQ (Université Paris-Sud, Laboratoire de Mathématiques d'Orsay, CNRS, UMR 8628 \\ et Institut Universitaire de France, Bâtiment 425, 91405 Orsay Cedex, France), \\ PATRICK GÉRARD (Université Paris-Sud, Laboratoire de Mathématiques d'Orsay, CNRS, UMR \\ 8628, Bâtiment 425, 91405 Orsay Cedex, France), \\ AND \\ NIKOLAY TZVETKOV (Université de Lille 1, Laboratoire Paul Painlevé, CNRS, UMR 8524, 59655 \\ Villeneuve d'Asq Cedex, France) \\ Dedicated to Walter Strauss for his 70th birthday, with our friendship and admiration

\begin{abstract}
We address the problem of describing solutions of the nonlinear Schrödin-
\end{abstract} \\ ger equation on a compact surface in the high frequency regime. In this context, we \\ introduce a nonnegative threshold, depending on the geometry of the surface, which can \\ be seen as a measurement of the nonlinear character of the equation, and we compute \\ this number for the torus and for the sphere, as a consequence of earlier arguments. \\ The last part is devoted to the study, on the sphere, of the critical regime associated to \\ this threshold. We prove that the effective dynamics are described by a new evolution \\ equation, the Resonant Hermite-Schrödinger equation.
}

1. Introduction. Let $(M, g)$ be a Riemannian manifold of dimension 2 without boundary. The (cubic) nonlinear Schrödinger equation on $M$ reads

$$
i \frac{\partial u}{\partial t}+\Delta u=|u|^{2} u
$$

where the unknown $u=u(t, x)$ is a complex-valued function on $\mathbb{R} \times M$. This equation firstly arises in Nonlinear Optics, where it is obtained as an envelope equation from an oscillatory regime of the Maxwell system with a nonlinear response (see e.g. Sulem-Sulem

Received December 31, 2008.

2000 Mathematics Subject Classification. Primary 35Q55; Secondary 35B30.

Key words and phrases. Nonlinear Schrödinger equations, Strichartz estimates, Propagation of oscillations, Spherical harmonics, Nonhomogeneous media.

E-mail address: nicolas.burq@math.u-psud.fr

E-mail address: patrick.gerard@math.u-psud.fr

E-mail address: nikolay.tzvetkov@math.univ-lille1.fr 
[20]). In this context, the inhomogeneity represented by the metric $g$ may be interpreted for instance as modelling a nonuniform optical index. Equation (1.1) also comes from Quantum Mechanics: indeed, the cubic Schrödinger equation in $\mathbb{R}^{3}$ is now commonly used in the theory of Bose-Einstein condensates and in the theory of superfluidity (see 12 for a mathematical justification). If a confining potential in normal directions to some surface of $\mathbb{R}^{3}$ is added, the asymptotics are expected to be given by equation (1.1) (see [1]).

From the mathematical point of view, equation (1.1) is a Hamiltonian system, associated to the energy

$$
H=\int_{M}\left(|\nabla u|^{2}+\frac{1}{2}|u|^{4}\right) d x
$$

which is therefore a conservation law of the $t$-evolution. Moreover, the invariance of $H$ by $u \mapsto \mathrm{e}^{i \theta} u$, with $\theta \in \mathbb{R}$, leads to a second conservation law,

$$
\|u(t)\|_{L^{2}(M)}=\text { cst . }
$$

Using these conservation laws, global existence and uniqueness of solutions to the Cauchy problem for (1.1) with data in the Sobolev space $H^{1}(M)$ rely on classical arguments, as we will recall below. Our goal in this paper will be rather to address the problem of describing the solution in the high frequency regime, depending on the size of the energy $H$ with respect to the $L^{2}$-norm. As we will see, this problem is intimately related to a quantitative evaluation of the nonlinear character of equation (1.1), and the answer depends on the geometry of $M$. In order to emphasize the role of the geometry, we will assume from now on that the manifold $M$ is compact.

\section{A brief review of the Cauchy problem.}

Theorem 2.1 (Brézis-Gallouët 4, Vladimirov 22]). For every $u_{0} \in H^{1}(M)$, there exists a unique solution $u \in C\left(\mathbb{R}, H^{1}(M)\right)$ of (1.1) with $u(0)=u_{0}$. Moreover, if $u_{0} \in H^{s}(M)$ for some $s>1$, then $u \in C\left(\mathbb{R}, H^{s}(M)\right)$.

Proof. We just give a sketch.

The local existence for data in $H^{s}(M)$, for $s>1$ is standard. The rest of the proof relies on the fact that the conservation laws control the $H^{1}$-norm, and that $H^{1}(M)$ is "almost" contained in $L^{\infty}(M)$ since $M$ is two dimensional. More precisely, globalization in $H^{s}, s>1$ is obtained by means of the inequality

$$
\|u\|_{L^{\infty}} \leq C_{s}\|u\|_{H^{1}}\left(\log \left(2+\frac{\|u\|_{H^{s}}}{\|u\|_{H^{1}}}\right)\right)^{\frac{1}{2}}, s>1,
$$

proved in [4] in the case of a smooth plane domain. The proof in the case of a compact manifold is similar.

As for data in $H^{1}$, global existence of weak solutions is just a compactness argument in view of the conservation laws. The hard part is uniqueness of these weak solutions, and it follows from the estimate

$$
\|u\|_{L^{p}} \leq C \sqrt{p}\|u\|_{H^{1}}, p<\infty,
$$

or from the associated Trudinger inequality (see [22] or Ogawa [17]). 
We are now in a position to address the problem of describing high frequency regimes for (1.1).

3. The case of bounded energy. We start with a property which expresses that the cubic 2D NLS evolution propagates oscillations with bounded energy as the linear Schrödinger equation.

Proposition 1. Let $\left(u_{0}^{n}\right)$ be a bounded sequence of $H^{1}(M)$, weakly convergent to 0 . Denote by $u^{n}$ the solution of $(N L S)$ with $u^{n}(0)=u_{0}^{n}$. Then

$$
u^{n}(t)=\mathrm{e}^{i t \Delta} u_{0}^{n}+o(1)
$$

in $H^{1}(M)$, uniformly for $t \in[-T, T], T>0$.

Remark 3.1. (1) The information claimed by Proposition 1 has important consequences in Control Theory (see [11).

(2) Notice that the methods used in the previous section to solve the Cauchy problem do not give such information.

Proof. From the Duhamel equation,

$$
u^{n}(t)=\mathrm{e}^{i t \Delta} u_{0}^{n}-i \int_{0}^{t} \mathrm{e}^{i\left(t-t^{\prime}\right) \Delta}\left(\left|u^{n}\left(t^{\prime}\right)\right|^{2} u^{n}\left(t^{\prime}\right)\right) d t^{\prime}
$$

we get

$$
\begin{aligned}
\left\|u^{n}(t)-\mathrm{e}^{i t \Delta} u_{0}^{n}\right\|_{H^{1}} & \leq C \int_{0}^{t}\left\|\left|u^{n}\left(t^{\prime}\right)\right|^{2} u^{n}\left(t^{\prime}\right)\right\|_{H^{1}} d t^{\prime} \leq C \int_{0}^{t}\left\|u^{n}\left(t^{\prime}\right)\right\|_{L^{\infty}}^{2}\left\|u^{n}\left(t^{\prime}\right)\right\|_{H^{1}} d t^{\prime} \\
& \leq C \int_{0}^{t}\left\|u^{n}\left(t^{\prime}\right)\right\|_{L^{\infty}(M)}^{2} d t^{\prime} \sup _{t^{\prime} \in[0, t]}\left\|u^{n}\left(t^{\prime}\right)\right\|_{H^{1}(M)} .
\end{aligned}
$$

Therefore the claim is proved if we establish that

$$
\int_{-T}^{T}\left\|u^{n}(t)\right\|_{L^{\infty}(M)}^{2} d t \rightarrow 0
$$

for every $T>0$. For this crucial step, we appeal to an important estimate for the linear Schrödinger equation obtained in Burq-Gérard-Tzvetkov [5], which can also be deduced from results in Staffilani-Tataru [19].

LEMMA 3.2 .

$$
\left(\int_{-T}^{T}\left\|\mathrm{e}^{i t \Delta} f\right\|_{L^{\infty}(M)}^{2} d t\right)^{\frac{1}{2}} \leq C_{s}\|f\|_{H^{s}(M)}, s>\frac{1}{2} .
$$

A consequence of this lemma is that, under the assumptions of Proposition 1 ,

$$
\int_{-T}^{T}\left\|\mathrm{e}^{i t \Delta} u_{0}^{n}\right\|_{L^{\infty}(M)}^{2} d t \rightarrow 0 .
$$

Moreover, combining Minkowski's inequality with the lemma, $\forall s>1 / 2$,

$$
\int_{-T}^{T}\left\|\int_{0}^{t} \mathrm{e}^{i\left(t-t^{\prime}\right) \Delta} F\left(t^{\prime}\right) d t^{\prime}\right\|_{L^{\infty}(M)}^{2} d t \leq C\|F\|_{L^{1}\left([-T, T], H^{s}(M)\right)}^{2} .
$$


Hence, from Duhamel's formula,

$$
\left\|u^{n}\right\|_{L^{2}\left([-T, T], L^{\infty}(M)\right)} \leq C\left(o(1)+\left\|u^{n}\right\|_{L^{2}\left([-T, T], L^{\infty}(M)\right)}^{2}\right),
$$

and the conclusion follows from a bootstrap argument on the time variable.

4. The case of high energy solutions. As a brief motivation to what follows, let us mention that many PDEs arising in physics and involving small parameters can be reduced, after some change of scale, to a fixed PDE with a sequence of oscillatory data. In order to generalize Proposition 1 to solutions with unbounded energy, we introduce the following definition.

Definition 4.1. Let $s \in(0,1]$, and let $\left(u^{n}\right)$ be a sequence of $H^{1}(M)$.

We shall say that $u^{n}$ is high frequency $H^{s}$-normalized if the following two conditions hold :

(1) $\left\|u^{n}\right\|_{L^{2}} \rightarrow 0$.

(2) $\left\|\nabla u^{n}\right\|_{L^{2}} \leq C\left\|u^{n}\right\|_{L^{2}}^{-\frac{1-s}{s}}$.

Notice that the above conditions imply that $u^{n}$ is bounded in $H^{s}$. Moreover, by the Sobolev imbedding, the second condition is equivalent to

$$
H\left(u^{n}\right) \leq C\left\|u^{n}\right\|_{L^{2}}^{-2 \frac{1-s}{s}}
$$

hence this property is preserved by the evolution of (1.1). We now introduce the main property which will allow us to evaluate the nonlinear character of equation (1.1).

Definition 4.2. We shall say that property $\mathcal{P}(s)$ holds on $M$ if, for every $H^{s}$ normalized high frequency Cauchy data $\left(u_{0}^{n}\right)$, the solution $u^{n}$ of

$$
i \partial_{t} u^{n}+\Delta u^{n}=\lambda\left|u^{n}\right|^{2} u^{n}, u^{n}(0)=u_{0}^{n}
$$

satisfies

$$
\left\|u^{n}(t)-\mathrm{e}^{i t \Delta} u_{0}^{n}\right\|_{H^{s}} \rightarrow 0
$$

uniformly in $\lambda \in] 0,1], t \in[-T, T], T>0$.

This definition calls for several comments. First of all, notice that property $\mathcal{P}(1)$ is the property proved in section 3. Secondly, since

$$
\int_{-T}^{T}\left\|\mathrm{e}^{i t \Delta} f\right\|_{L^{\infty}(M)}^{2} d t \leq C_{s}\|f\|_{H^{s}}^{2}, s>\frac{1}{2},
$$

by the same arguments as in Proposition 1, we infer that $\mathcal{P}(s)$ holds for every $s>\frac{1}{2}$.

Finally, we claim that

$$
\mathcal{P}(s) \Rightarrow \mathcal{P}\left(s^{\prime}\right), \forall s^{\prime} \in(s, 1) .
$$

Indeed, if $u^{n}$ is $H^{s^{\prime}}$-normalized and solves

$$
i \partial_{t} u^{n}+\Delta u^{n}=\lambda\left|u^{n}\right|^{2} u^{n}
$$

then $\tilde{u}^{n}:=\left\|u_{0}^{n}\right\|_{L^{\frac{s-s^{\prime}}{s^{\prime}}}}^{\frac{s^{\prime}}{n}}$ is $H^{s}$-normalized and solves

$$
i \partial_{t} \tilde{u}^{n}+\Delta \tilde{u}^{n}=\mu_{n}\left|\tilde{u}^{n}\right|^{2} \tilde{u}^{n},
$$

with

$$
\mu_{n}=\left\|u_{0}^{n}\right\|_{L^{2}}^{\frac{\left(s^{\prime}-s\right)}{s^{\prime}}} \lambda \leq \lambda,
$$


for $n$ large enough. The claim follows from the application of $\mathcal{P}(s)$ to $\tilde{u}^{n}$. Notice that the somewhat technical introduction of the parameter $\lambda$ in Definition 4.2 is justified by property (4.1).

At this stage, it is natural to introduce the following quantity, for a given surface $(M, g)$,

$$
s_{c}(M, g)=\inf \{s \in(0,1): \mathcal{P}(s) \text { holds on } M\} .
$$

Notice that $s_{c}(M, g)$ is a measurement of how much equation (1.1) is nonlinear: indeed, the smaller $s_{c}(M)$ is, the higher energy oscillations are linearly propagated by (1.1). From the above observations, we already know that

$$
s_{c}(M, g) \leq \frac{1}{2},
$$

for every $M$. However, it turns out that the exact value of $s_{c}(M, g)$ depends on the geometry of $(M, g)$. The next theorem gives the answer for two well-known compact surfaces.

THEOREM 4.3. With the standard metrics, we have

$$
\begin{gathered}
s_{c}\left((\mathbb{R} / 2 \pi \mathbb{Z})^{2}\right)=0, \\
s_{c}\left(S^{2}\right)=\frac{1}{4} .
\end{gathered}
$$

Moreover, $\mathcal{P}(1 / 4)$ does not hold on $S^{2}$.

The part of Theorem 4.3 concerning the torus is a consequence of arguments developed by Bourgain in $\left[3\right.$. Notice that $s_{c}(\mathbb{R} / a \mathbb{Z} \times \mathbb{R} / b \mathbb{Z})$ is still unknown for arbitrary values of $a, b$, though it has been proved to be $\leq 1 / 3$ by Catoire-Wang in [10. The part of Theorem 4.3 concerning the sphere is deduced from arguments by Burq-Gérard-Tzvetkov in [6] and [7] (see also [13]). A generalization of these arguments shows that, if $M$ is a surface of revolution with a nondegenerate equator, $s_{c}(M) \geq 1 / 4$, but the upper bound is still open. Other generalizations of this type can be found in Thomann 21.

Notice that the value $1 / 4$ of $s_{c}\left(S^{2}\right)$ may seem surprising in view of the observation that the threshold $s=1 / 2$ in Lemma 3.2 is optimal on $S^{2}$. In other words, the estimate

$$
\left(\int_{-T}^{T}\left\|\mathrm{e}^{i t \Delta} f\right\|_{L^{\infty}\left(S^{2}\right)}^{2} d t\right)^{\frac{1}{2}} \leq C_{s}\|f\|_{H^{s}\left(S^{2}\right)}
$$

fails if $s<\frac{1}{2}$. Notice that the similar one on $\mathbb{R}^{2}$ holds $\forall s>0$ (see Ginibre-Velo [14]). To prove the failure of the estimate, take for $f$ the zonal spherical harmonic $k_{n}\left(x_{0},.\right)$ of degree $n$ at some point $x_{0}$ of $S^{2}$, characterized by

$$
H\left(x_{0}\right)=\int_{S^{2}} k_{n}\left(x_{0}, x\right) H(x) d x
$$

for every spherical harmonic $H$ of degree $n$. It is easy to check that

$$
k_{n}\left(x_{0}, x\right)=\sum_{j=1}^{2 n+1} \overline{e_{j}\left(x_{0}\right)} e_{j}(x),
$$


where $\left(e_{j}\right)$ is any orthonormal basis of spherical harmonics of degree $n$. Then, using the invariance by rotation, we have

$$
k_{n}\left(R x_{0}, R x\right)=k_{n}\left(x_{0}, x\right)
$$

for every rotation $R$ of $S^{2}$ and therefore $k_{n}\left(x_{0}, x_{0}\right)$ does not depend on $x_{0}$. Consequently,

$$
\left|k_{n}\left(x_{0}, x_{0}\right)\right|=\sum_{j=1}^{2 n+1}\left|e_{j}\left(x_{0}\right)\right|^{2}=2 n+1=\left\|k_{n}\left(x_{0}, .\right)\right\|_{L^{2}}^{2},
$$

and on the other hand, by the Cauchy-Schwarz inequality,

$$
\left\|k_{n}\left(x_{0}, .\right)\right\|_{L^{\infty}}=\sum_{j=1}^{2 n+1}\left|e_{j}\left(x_{0}\right)\right|^{2}=k_{n}\left(x_{0}, x_{0}\right)
$$

so that

$$
\left(\int_{-T}^{T}\left\|\mathrm{e}^{i t \Delta} k_{n}\left(x_{0}, .\right)\right\|_{L^{\infty}\left(S^{2}\right)}^{2} d t\right)^{\frac{1}{2}}=\sqrt{T}\left\|k_{n}\left(x_{0}, .\right)\right\|_{L^{\infty}}=\sqrt{T}(2 n+1),
$$

while

$$
\left\|k_{n}\left(x_{0}, .\right)\right\|_{H^{s}} \simeq n^{s+1 / 2},
$$

which, if $n$ tends to $\infty$, contradicts inequality (4.2) for $s<1 / 2$.

This observation, compared to Theorem 4.3 shows that the approach we used in Section 3 to estimate the solutions of (1.1) is not the optimal one. In the next two sections, we shall briefly present a better approach, due to Bourgain, and then explain the value $1 / 4$ on $S^{2}$. Finally, in the last section, we shall investigate the effective nonlinear dynamics on $S^{2}$ of a class of $H^{1 / 4}$ normalized data.

\section{The use of bilinear Strichartz inequalities.}

Definition 5.1. Let $s_{0} \geq 0$. We shall say that a bilinear Strichartz inequality of order $s_{0}$ holds on $M$ if

$$
\left\|\mathrm{e}^{i t \Delta} f \mathrm{e}^{i t \Delta} g\right\|_{L^{2}\left([0,1]_{t} \times M\right)} \leq C N^{s_{0}}\|f\|_{L^{2}(M)}\|g\|_{L^{2}(M)},
$$

where $N \leq L$ are arbitrary positive integers and $f, g$ are arbitrary functions on $M$ such that

$$
f=\mathbf{1}_{\sqrt{-\Delta} \sim N} f, g=\mathbf{1}_{\sqrt{-\Delta} \sim L} g .
$$

Notice that estimate (5.1) implies the following Strichartz inequality:

$$
\left\|\mathrm{e}^{i t \Delta} f\right\|_{L^{4}\left([0,1]_{t} \times M\right)} \leq C\|f\|_{H^{s_{0} / 2}(M)},
$$

but that it has the additional advantage of giving a better estimate in the case of interaction of high frequency terms with lower frequency terms. This estimate was proved in [7] to be a criterion for the existence of a local-in-time smooth flow map for (1.1) on $H^{s_{0}+\varepsilon}(M)$.

The following theorem can be deduced from arguments in 7 .

Theorem 5.2. If a bilinear Strichartz inequality of order $s_{0}$ holds on $M$, then, for every $s>s_{0}, \mathcal{P}(s)$ holds on $M$. 
Let us give some indications for the proof, referring to 7 for missing details. Following Bourgain [3], we introduce the norms $\|u\|_{X_{T}^{s, b}}=\left\|\mathrm{e}^{-i t \Delta} u(t)\right\|_{H^{b}\left([0, T]_{t}, H^{s}(M)\right)}$, and we observe that $X_{T}^{s, b} \subset C\left([0, T], H^{s}\right)$ as soon as $b>1 / 2$. Then we replace the estimate

$$
\sup _{0 \leq t \leq T}\left\|\int_{0}^{t} \mathrm{e}^{i\left(t-t^{\prime}\right) \Delta} F\left(t^{\prime}\right) d t^{\prime}\right\|_{H^{s}} \leq C\|F\|_{L^{1}\left([0, T], H^{s}\right)}
$$

used in section 3 by the estimate

$$
\left\|\int_{0}^{t} \mathrm{e}^{i\left(t-t^{\prime}\right) \Delta} F\left(t^{\prime}\right) d t^{\prime}\right\|_{X_{T}^{s, b}} \leq C T^{1-b-b^{\prime}}\|F\|_{X_{T}^{s,-b^{\prime}}}
$$

if $b>\frac{1}{2}>b^{\prime}, b+b^{\prime}<1, T \leq 1$. Notice that the latter estimate is nothing but an inequality about antiderivation in Sobolev spaces on the time interval $(0, T)$.

The main step is then the following observation: (5.1) implies, for $s \geq \sigma>s_{0}$ and some $b^{\prime}<\frac{1}{2}$, that

$$
\left\||u|^{2} u\right\|_{X_{T}^{s,-b^{\prime}}} \leq C\|u\|_{X_{T}^{\sigma, b^{\prime}}}^{2}\|u\|_{X_{T}^{s, b^{\prime}}}
$$

If $u_{0}^{n}$ is a high frequency $H^{s}$ normalized sequence, denote by $u^{n}$ the solution of (1.1) with $u^{n}(0)=u_{0}^{n}$, and by $v^{n}$ the solution of the linear Schrödinger equation with the same Cauchy data $u_{0}^{n}$. We have

$$
u^{n}(t)=v^{n}(t)-i \int_{0}^{t} \mathrm{e}^{i\left(t-t^{\prime}\right) \Delta}\left(\left|u^{n}\left(t^{\prime}\right)\right|^{2} u^{n}\left(t^{\prime}\right)\right) d t^{\prime} .
$$

Consequently, for $\sigma<s$ and $b^{\prime}<1 / 2$,

$$
\left\|u^{n}\right\|_{X_{T}^{\sigma, b^{\prime}}} \leq\left\|v^{n}\right\|_{X_{T}^{\sigma, b^{\prime}}}+C\left\|\left.|| u^{n}\right|^{2} u^{n}\right\|_{X_{T}^{\sigma,-b^{\prime}}} \leq C\left(\left\|u_{0}^{n}\right\|_{H^{\sigma}}+\left\|u^{n}\right\|_{X_{T}^{\sigma, b^{\prime}}}^{3}\right) .
$$

From the assumptions, we know that $\left\|u_{0}^{n}\right\|_{H^{\sigma}}$ tends to 0 . Hence, by a bootstrap argument, we conclude that

$$
\left\|u^{n}\right\|_{X_{T}^{\sigma, b^{\prime}}} \rightarrow 0
$$

for every $T \leq 1$; notice that, since $b^{\prime}<1 / 2,\|u\|_{X_{T}^{\sigma, b^{\prime}}}$ tends to 0 as $T$ tends to 0 .

We now come back to the Duhamel formula (5.3) and we estimate the difference $u^{n}-v^{n}$,

$$
\left\|u^{n}-v^{n}\right\|_{X_{T}^{s, b}} \leq C\left\|\left|u^{n}\right|^{2} u^{n}\right\|_{X_{T}^{s,-b^{\prime}}} \leq C\left\|u^{n}\right\|_{X_{T}^{\sigma, b^{\prime}}}^{2}\left\|u^{n}\right\|_{X_{T}^{s, b^{\prime}}} .
$$

The proof will therefore be complete if we establish that $\left\|u^{n}\right\|_{X_{T}^{s, b^{\prime}}}$ is bounded, which is still a consequence of similar estimates and of the bootstrap lemma, using this time the smallness of the factor $T^{1-b-b^{\prime}}$ if $T$ is small in (5.2).

We turn to the case of $S^{2}$. In order to apply Theorem 5.2, we need to establish bilinear Strichartz estimates on $S^{2}$. Testing (5.1) with $f=g=\varphi_{n}$ (spherical harmonic of degree $n)$, we get the following necessary condition:

$$
\left\|\varphi_{n}\right\|_{L^{4}} \leq C n^{\frac{s_{0}}{2}}\left\|\varphi_{n}\right\|_{L^{2}}
$$

which imposes $s_{0} \geq \frac{1}{4}$ in view of the example of the highest weight harmonic,

$$
\varphi_{n}\left(x_{1}, x_{2}, x_{3}\right)=\left(x_{1}+i x_{2}\right)^{n} .
$$

It is known since results by Sogge [18, that the growth $n^{1 / 8}$ is the worst possible in the above eigenfunction estimate. It is in fact possible to prove a bilinear version of this 
result (see [7], [8], [9]), and, combining with the clustering properties of the spectrum, one can prove that (5.1) holds on $S^{2}$ for every $s_{0}>\frac{1}{4}$, which proves that

$$
s_{c}\left(S^{2}\right) \leq \frac{1}{4}
$$

In order to prove the reverse inequality, we need a nonlinear version of the highest weight harmonic (5.4).

6. The failure of $\mathcal{P}(1 / 4)$ on $S^{2}$. We denote by $R_{\alpha}$ the rotation of the angle around the $x_{3}$-axis in $\mathbb{R}^{3}$, and we define

$$
H_{n}^{s}\left(S^{2}\right):=\left\{u \in H^{s}\left(S^{2}\right): \forall \alpha \in \mathbb{R}, u\left(R_{\alpha} x\right)=\mathrm{e}^{i n \alpha} u(x)\right\} .
$$

Notice that, up to a meaningless multiplicative constant, the function $\varphi_{n}$ defined by (5.4) is a minimizer for the problem

$$
\min \left\{\int_{S^{2}}|\nabla \varphi(x)|^{2} d x, \varphi \in H_{n}^{1}\left(S^{2}\right),\|\varphi\|_{L^{2}\left(S^{2}\right)}=n^{-\frac{1}{4}}\right\} .
$$

Similarly, let $\psi_{n}$ be a minimizer of

$$
\min \left\{\int_{S^{2}}|\nabla \psi(x)|^{2}+\frac{1}{2}|\psi(x)|^{4} d x, \psi \in H_{n}^{1}\left(S^{2}\right),\|\psi\|_{L^{2}\left(S^{2}\right)}=n^{-\frac{1}{4}}\right\} .
$$

Then the Euler-Lagrange equation reads

$$
-\Delta \psi_{n}+\left|\psi_{n}\right|^{2} \psi_{n}=\omega_{n} \psi_{n}
$$

so that

$$
u^{n}(t)=\mathrm{e}^{-i t \omega_{n}} \psi_{n}
$$

is a solution of (1.1), which defines a high frequency $H^{1 / 4}$ normalized sequence. One can prove (see [13]) that, for some $\theta_{n}$,

$\left\|\psi_{n}-\mathrm{e}^{i \theta_{n}} \varphi_{n}\right\|_{H^{1 / 4}}=o(1), \omega_{n}=\frac{1}{\left\|\varphi_{n}\right\|_{L^{2}}^{2}}\left(\left\|\nabla \varphi_{n}\right\|_{L^{2}}^{2}+\left\|\varphi_{n}\right\|_{L^{4}}^{4}\right)+o(1)=n(n+1)+\gamma+o(1)$,

where

$$
\gamma:=\lim _{n \rightarrow \infty} \frac{\left\|\varphi_{n}\right\|_{L^{4}}^{4}}{\left\|\varphi_{n}\right\|_{L^{2}}^{2}}>0
$$

Hence

$$
\left\|u^{n}(t)-\mathrm{e}^{i t \Delta} u^{n}(0)\right\|_{H^{1 / 4}} \rightarrow\left|\mathrm{e}^{i \gamma t}-1\right|,
$$

which contradicts $\mathcal{P}(1 / 4)$.

Let us mention that the use of stationary solutions for proving instability of the flow map of a nonlinear dispersive equation already arose in papers by Birnir-Kenig-PonceSvanstedt-Vega [2] and Kenig-Ponce-Vega [16]. A slightly different proof can be found in [6]. 
7. The effective dynamics on $H_{n}^{1 / 4}\left(S^{2}\right), n \rightarrow \infty$. In this section, we describe the effective dynamics corresponding to the concentration on the equator which is responsible for the failure of $\mathcal{P}(1 / 4)$, according to the previous section. Let $u_{0}^{n} \in H_{n}^{1}\left(S^{2}\right)$ satisfying

$$
\left\|u_{0}^{n}\right\|_{L^{2}} \leq C n^{-1 / 4},\left\|\nabla u_{0}^{n}\right\|_{L^{2}}^{2}-n(n+1)\left\|u_{0}^{n}\right\|_{L^{2}}^{2} \leq C n^{1 / 2} .
$$

These bounds clearly imply that $\left(u_{0}^{n}\right)$ is a high frequency $H^{1 / 4}$-normalized sequence. We would like to describe the solution $u^{n}(t)$ of (1.1) with $u^{n}(0)=u_{0}^{n}$, up to a small error in $H^{1 / 4}\left(S^{2}\right)$ as $n \rightarrow \infty$. Let us expand $u_{0}^{n}$ in spherical harmonics as

$$
u_{0}^{n}(x)=n^{-1 / 4} \sum_{k=0}^{\infty} z_{k, 0}^{n} h_{n, k}(x)
$$

where $h_{n, k}$ is the $L^{2}$-normalized harmonic of degree $n+k$ in $H_{n}^{1}$.

If $H_{k}$ denotes the Hermite function normalized in $L^{2}(\mathbb{R})$, one can prove that, as $n$ goes to $\infty$,

$$
h_{n, k}(x)=n^{\frac{1}{4}} \mathrm{e}^{i n \theta} H_{k}\left(\sqrt{n} x_{3}\right)+o(1),\left(x_{1}+i x_{2}\right):=\sqrt{1-x_{3}^{2}} \mathrm{e}^{i \theta} .
$$

These asymptotics can be seen as an illustration of the general principle of single well approximation; see e.g. Helffer [15].

For $\tau \in \mathbb{T}:=\mathbb{R} / 2 \pi \mathbb{Z}$ and $y \in \mathbb{R}$, we introduce the following concentration profile:

$$
U_{0}(\tau, y)=\sum_{k=0}^{\infty} z_{k, 0} \mathrm{e}^{-i k \tau} H_{k}(y)
$$

where $z_{k, 0}:=\lim _{n \rightarrow \infty} z_{k, 0}^{n}$ (well-defined up to the extraction of a subsequence), so that

$$
u_{0}^{n}(x)-\mathrm{e}^{i n \theta} U_{0}\left(0, \sqrt{n} x_{3}\right) \rightarrow 0 \text { in } H^{1 / 4}\left(S^{2}\right) .
$$

Notice that, in view of the assumptions (7.1),

$$
\sum_{k=0}^{\infty} k\left|z_{k, 0}\right|^{2}=\left(i \partial_{\tau} U_{0}, U_{0}\right)_{L^{2}}<\infty,
$$

and that $U_{0}$ satisfies the following linear equation on $\mathbb{T} \times \mathbb{R}$ :

$$
i \frac{\partial U_{0}}{\partial \tau}+\frac{1}{2}\left(\frac{\partial^{2}}{\partial y^{2}}-y^{2}+1\right) U_{0}=0 .
$$

Denote by $P$ the orthogonal projector onto the nullspace $\mathcal{N}$ of

$$
i \frac{\partial}{\partial \tau}+\frac{1}{2}\left(\frac{\partial^{2}}{\partial y^{2}}-y^{2}+1\right)
$$

in $L^{2}\left(\mathbb{T}_{\tau} \times \mathbb{R}_{y}\right)$. The following theorem describes the evolution with $t$ of the concentration profile of $u^{n}(t)$.

TheOrem 7.1 (Burq-Gérard-Tzvetkov, 2007). Let $U=U(t, \tau, y)$ be the solution of the following equation:

$$
\left(i \frac{\partial U}{\partial t}+\frac{\partial^{2} U}{\partial \tau^{2}}\right)=P\left(|U|^{2} U\right),
$$


with the Cauchy data $U(0, \tau, y)=U_{0}(\tau, y)$. Then, $\forall T>0$, we have, in $H^{1 / 4}$, uniformly for $t \in[-T, T]$,

$$
u^{n}(t, x)=\mathrm{e}^{i n \theta-i n(n+1) t} U\left(t,(2 n+1) t, \sqrt{n} x_{3}\right)+o(1) .
$$

Equation (7.2) is obtained by extracting the resonant part of the infinite system on the unknown $\left(z_{k}^{n}\right)_{k \geq 0}$. The projection on this resonant part is provided by operator $P$. One can call (7.2) the Resonant Hermite-Schrödinger equation. It is a Hamiltonian system with three conservation laws,

$$
Q=\|U\|_{L^{2}(\mathbb{T} \times \mathbb{R})}^{2}, M=\left(i \partial_{\tau} U, U\right)_{L^{2}}, E=\left\|\partial_{\tau} U\right\|_{L^{2}}^{2}+\frac{1}{2}\|U\|_{L^{4}}^{4} .
$$

Moreover, the combination of dispersive estimates for the Schrödinger equation on $\mathbb{T}$ (see [3]) with the bilinear estimates for the Hermite functions,

$$
\left\|H_{n} H_{\ell}\right\|_{L^{2}(\mathbb{R})} \leq \frac{C_{\varepsilon}}{(1+\max (n, \ell))^{\frac{1}{4}-\varepsilon}},
$$

leads to the following well-posedness result for rough data.

Theorem 7.2 (Burq-Gérard-Tzvetkov, 2008). Equation (7.2) is globally well-posed on $\mathcal{N}$, and the flow map is continuous for some negative Sobolev norm.

The proofs of Theorems 7.1 and 7.2 will appear in a paper in preparation.

Let us conclude with some comments and open questions.

- In the particular case of one initial mode,

$$
U_{0}(\tau, y)=z_{p, 0} \mathrm{e}^{-i p \tau} H_{p}(y)
$$

it is easy to check that

$$
U(t, \tau, y)=\mathrm{e}^{-i\left(p^{2}+c_{p}\left|z_{p, 0}^{n}\right|^{2}\right) t} z_{p, 0} \mathrm{e}^{-i p \tau} H_{p}(y),
$$

which means that no other mode appears. This observation, combined with Theorem 7.1 includes the nonlinear highest weight harmonic approximation of the previous section and the result of $[6]$.

- As soon as the Cauchy data involve two different modes, Theorem 7.1 asserts that the solution instantaneously activates all the other modes. This answers a question asked to us by J. Rauch and Y. Tsutsumi.

- For $s<1 / 4$, effective dynamics in $H^{s}$ are expected to be described by large time behavior of the equation (7.2). This leads to very delicate stability issues, where the study of invariant tori for the Hamiltonian system (7.2) might play a central role.

\section{REFERENCES}

[1] Ben Abdallah, Naoufel; Méhats, Florian; Schmeiser, Christian; Weishäupl, Rada M., The nonlinear Schrödinger equation with a strongly anisotropic harmonic potential. SIAM J. Math. Anal. 37 (2005), no. 1, 189-199 (electronic). MR2176928(2006f:35257)

[2] Birnir, B., Kenig, C., Ponce, G., Svanstedt, N., Vega, L., On the ill-posedness of the IVP for the generalized KdV and nonlinear Schrödinger equation. J. London Math. Soc. 53 (1996), 551-559. MR 1396718 (97d:35233) 
[3] Bourgain, J., Fourier transform restriction phenomena for certain lattice subsets and applications to nonlinear evolution equations. I. Schrödinger equations. Geom. and Funct. Anal. 3 (1993), 107156. MR1209299 (95d:35160a)

[4] Brézis, H., Gallouët, T., Nonlinear Schrödinger evolution equations. Nonlinear Anal. 4 (1980), 677-681. MR582536 (81i:35139)

[5] Burq, N., Gérard, P., Tzvetkov, N., Strichartz inequalities and the nonlinear Schrödinger equation on compact manifolds. Amer. J. Math. 126 (2004), 569-605. MR2058384 (2005h:58036)

[6] Burq, N., Gérard, P., Tzvetkov, N., An instability property of the nonlinear Schrödinger equation on $S^{d}$. Math. Res. Lett. 9 (2002), 323-335. MR1909648 (2003c:35144)

[7] Burq, N., Gérard, P., Tzvetkov, N., Bilinear eigenfunction estimates and the nonlinear Schrödinger equation on surfaces. Invent. Math. 159 (2005), 187-223. MR2142336 (2005m:35275)

[8] Burq, N., Gérard, P., Tzvetkov, N., Multilinear eigenfunction estimates and global existence for the three dimensional nonlinear Schrödinger equations. Ann. Scient. École Norm. Sup. 38 (2005), 255-301. MR 2144988 (2006m:35337)

[9] Burq, N., Gérard, P., Tzvetkov, N., The Cauchy Problem for the nonlinear Schrödinger equation on compact manifolds. In Phase Space Analysis of Partial Differential Equations (ed. by F. Colombini and L. Pernazza), vol.I. Centro di Ricerca Matematica Ennio de Giorgi, Scuola Normale Superiore, Pisa, 2004, 21-52. MR2144405 (2006b:35297)

[10] Catoire, F., Wang, W.-M., Bounds on Sobolev norms for the nonlinear Schrödinger equation on general tori. Preprint, September 2008, http://arxiv.org/abs/0809.4633.

[11] Dehman, B., Gérard, P., Lebeau, G., Stabilization and Control for the Nonlinear Schrödinger Equation on a Compact Surface. Mathematische Zeitschrift 254, (2006), 729-749. MR2253466 (2007g:93007)

[12] Erdös, L., Schlein, B., Yau, H. -T., Derivation of the cubic non-linear Schrödinger equation from quantum dynamics of many-body systems. Invent. Math. 167 (2007), 515-614. MR2276262 $(2007 \mathrm{~m}: 81258)$

[13] Gérard, P., Nonlinear Schrödinger equations on compact manifolds. In European Congress of Mathematics, Stockholm, June 27-July 2, 2004 (ed. by Ari Laptev). European Mathematical Society, Zürich, 2005, 121-139. MR2185741 (2006g:58057)

[14] Ginibre, J., Velo, G., The global Cauchy problem for the nonlinear Schrödinger equation revisited. Ann. Inst. H. Poincaré-Anal. Non Linéaire 2 (1985) 309-327. MR801582 (87b:35150)

[15] Helffer, B., Introduction to the semi-classical Analysis for the Schrödinger operator and applications. Lecture Notes in Mathematics 1336, Springer-Verlag (1986). MR.960278 (90c:81043)

[16] Kenig, C., Ponce, G., Vega, L., On the ill-posedness of some canonical dispersive equations. Duke Math. J. 106 (2001), 617-633. MR1813239 (2002c:35265)

[17] Ogawa, T., A proof of Trudinger's inequality and its application to nonlinear Schrödinger equations. Nonlinear Anal. 14 (1990), no. 9, 765-769. MR.1049119 (91d:35203)

[18] Sogge, C., Oscillatory integrals and spherical harmonics. Duke Math. Jour. 53, (1986), 43-65. MR.835795 (87g:42026)

[19] Staffilani, G., Tataru, D., Strichartz estimates for a Schrödinger operator with nonsmooth coeffcients. Comm. Partial Differential Equations 27, (2002), 1337-1372. MR1924470 (2003f:35248)

[20] Sulem, C., Sulem, P.-L., The Nonlinear Schrödinger Equation. Self-Focusing and Wave Collapse. Applied Mathematical Sciences, 139, Springer-Verlag, New York, 1999. MR1696311 (2000f:35139)

[21] Thomann, L., The WKB method and geometric instability for nonlinear Schrödinger equations on surfaces. Bull. Soc. Math. France 136 (2008), no. 2, 167-193. MR2415340

[22] Vladimirov, M. V., On the solvability of a mixed problem for a nonlinear Schrödinger equation of mixed type. Sov. Math. Dokl. 29 (1984), 281-284. MR0745511 (85h:35213) 